\title{
Oncologic Outcomes of Extended Lymphadenectomy without Liver Resection for T1/T2 Gallbladder Cancer
}

\author{
Jae Uk Chong ${ }^{1,2}$ and Woo Jung Lee ${ }^{1,3}$ \\ ${ }^{1}$ Division of Hepatobiliary and Pancreatic Surgery, Department of Surgery, Yonsei University College of Medicine, Seoul; \\ ${ }^{2}$ Department of Surgery, National Health Insurance Service Ilsan Hospital, Goyang; \\ ${ }^{3}$ Pancreatobiliary Cancer Center, Yonsei Cancer Center, Severance Hospital, Seoul, Korea.
}

\begin{abstract}
Purpose: This study provides a standardized operative strategical algorithm that can be applied to patients with T1/T2 gallbladder cancer (GBC). Our aim was to determine the oncologic outcome of radical cholecystectomy with para-aortic lymph node dissection without liver resection in $\mathrm{T} 1 / \mathrm{T} 2 \mathrm{GBC}$.

Materials and Methods: From January 2005 to December 2017, 164 patients with GBC underwent operations by a single surgeon at Severance Hospital. A retrospective review was performed for 113 of these patients, who were pathologically determined to be in stages $\mathrm{T} 1$ and $\mathrm{T} 2$ according to American Joint Committee on Cancer 7th guidelines.

Results: Of the 113 patients, 109 underwent curative resection for T1/T2 GBC; four patients who underwent palliative operations without radical cholecystectomies were excluded from further analyses. For all T1b and T2 lesions, radical cholecystectomy with para-aortic lymph node dissection was performed without liver resection. There were four GBC-related mortalities, and 5-year disease-specific survival was $97.0 \%$. The median follow-up was 50 months (range: 5-145 months). In all T stages, the median was not reached for survival analysis. Five-year disease-specific survival for T1a, T1b, and T2 were $100 \%, 94.1 \%$, and $97.1 \%$, respectively. Five-year disease-free survival for T1a, T1b, and T2 were 100\%, 87.0\%, and $91.8 \%$, respectively.

Conclusion: Our results suggest that the current operative protocol can be applied to minimal invasive operations for GBC with similar oncologic outcomes as open approach. For T1/T2 GBC, radical cholecystectomy, including para-aortic lymph node dissection, can be performed safely with favorable oncologic outcomes.
\end{abstract}

Key Words: Gallbladder neoplasms, cholecystectomy, lymph node dissection, survival analysis

\section{INTRODUCTION}

Gallbladder cancer (GBC) is a relatively rare disease that has been traditionally considered an incurable malignancy, owing to the advanced stage at diagnosis. ${ }^{1,2}$ Currently, radical surgery is the only curative option for GBC, but the extent of opera-

Received: February 11, 2019 Revised: October 15, 2019

Accepted: October 22, 2019

Corresponding author: Woo Jung Lee, MD, PhD, Division of Hepatobiliary and Pancreatic Surgery, Department of Surgery, Yonsei University College of Medicine, 50-1 Yonsei-ro, Seodaemun gu, Seoul 03722, Korea.

Tel: 82-2-2228-2120, Fax: 82-2-313-8289, E-mail: wjlee@yuhs.ac

-The authors have no potential conflicts of interest to disclose.

(C) Copyright: Yonsei University College of Medicine 2019

This is an Open Access article distributed under the terms of the Creative Commons Attribution Non-Commercial License (https://creativecommons.org/licenses/ by-nc/4.0) which permits unrestricted non-commercial use, distribution, and reproduction in any medium, provided the original work is properly cited. tion for T1/T2 GBC remains controversial. Generally, simple cholecystectomy alone (including laparoscopic cholecystectomy) is considered adequate for the pathologic stage of Tla GBC, as reflected by the current National Comprehensive Cancer Network guidelines (version 2017). However, for GBC of stage $\mathrm{Tl} \mathrm{b}$ or greater, radical cholecystectomy is recommended, which includes en bloc hepatic resection (segments IVB and V) and lymphadenectomy to clear all lymph nodes in the porta hepatis with/without bile duct resection. Nevertheless, the surgical extent for T1b and T2 GBC has been highly debated, and evidence of increased survival after radical cholecystectomy in patients with T1b GBC is lacking. ${ }^{3}$ Moreover, guidelines for surgical extent also vary greatly among institutions, and the global prevalence of GBC is imbalanced with high incidence rates reported in South America and Asia. ${ }^{4}$ However, the higher recurrence rate reported with simple cholecystectomy in cases with stage Tlb suggests that more radical operations should 
be considered. ${ }^{5}$

With regard to the extent of radical cholecystectomy, the policy for T1/T2 GBC at Severance Hospital traditionally included liver resection. However, our earlier experiences showed that recurrences in the liver rarely occur, and we opted not to resect the liver during radical cholecystectomy. ${ }^{6}$ We encountered recurrences in distant lymph nodes, especially para-aortic lymph nodes after radical cholecystectomy; therefore, beginning in 2005, we routinely extended lymphadenectomies to include the para-aortic lymph nodes in patients with $\mathrm{T} 1 / \mathrm{T} 2$ GBC. Moreover, our experiences with complications in the common bile duct after radical cholecystectomy have led us to include the insertion of a T-tube as the final step of operation. The present study analyzes the oncologic outcomes of patients at Severance Hospital undergoing these treatments with extended lymphadenectomy and without liver resection.

\section{MATERIALS AND METHODS}

\section{Patients and study design}

From January 2005 to December 2017, 164 patients with GBC underwent operation by a single surgeon (WJL) at Severance Hospital. We retrospectively reviewed the cases of 113 patients who underwent extended cholecystectomy without hepatectomy and were pathologically determined to be at stages $\mathrm{Tl}$ and T2. Pathological results, including $\mathrm{T}$ and $\mathrm{N}$ stages, were defined according to the American Joint Committee on Cancer (AJCC) cancer staging manual, 7th edition. Patients who underwent additional hepatectomy under the suspicion of T3 tumor were excluded as they were not within our treatment algorithm. Four patients who underwent palliative operations without radical cholecystectomy were also excluded. This study was reviewed and approved by the Institutional Review Board of Yonsei University College of Medicine, Seoul, Republic of Korea (IRB: 4-2017-0262).

\section{Preoperative evaluation}

Patients with suspected GBC from abdominal ultrasonography and/or computed tomography underwent positron emission tomography to assess distant metastases. Endoscopic ultrasonography was used to assess tumor invasion depth. In cases with incidentally diagnosed GBC, positron emission tomography was performed after laparoscopic cholecystectomy to evaluate possible metastatic lesions. Carbohydrate antigen 19-9 was evaluated as the tumor marker.

\section{Operative strategy and adjuvant treatment}

A schematic detailing the treatment algorithm is shown in Fig. 1. Three different approaches to radical cholecystectomy were employed: open or minimally invasive (laparoscopic and robotic). If preoperative evaluation suggested a T2 lesion with suspicious lymph node metastasis, the operation was per-

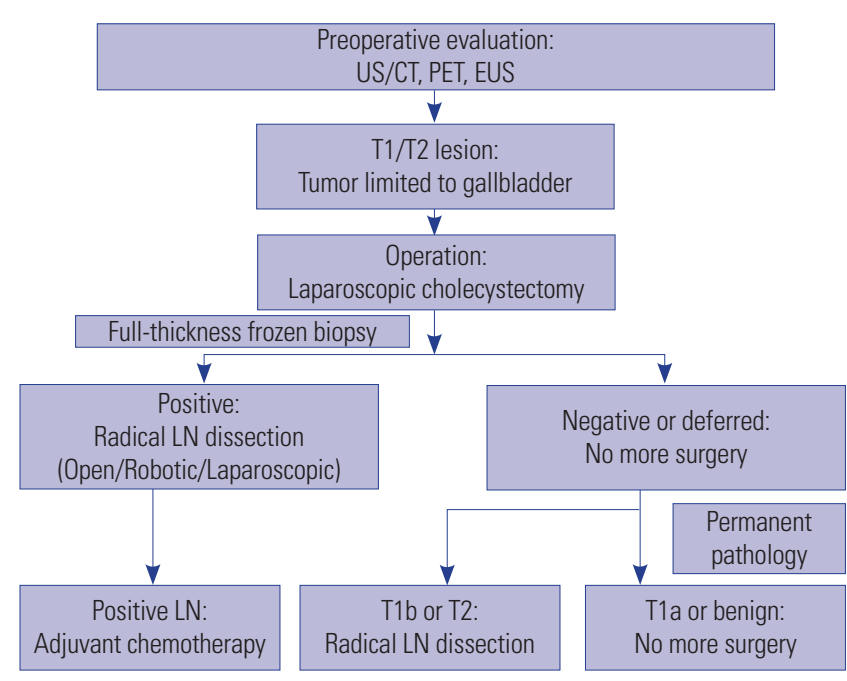

Fig. 1. Treatment algorithm for gallbladder carcinoma. US, ultrasonography; CT, computed tomography; PET: positron emission tomography; EUS, endoscopic ultrasonography; LN, lymph node.

formed using an open approach. For T1 and T2 lesions without suspicious lymph node metastasis, laparoscopic cholecystectomy was performed initially. During cholecystectomy, the cystic plate was completely removed with the gallbladder to avoid exposing the subserosal layer. If the results of a full-thickness frozen biopsy were positive for malignancy, radical lymph node dissection was performed. The extents of lymphadenectomy were hepatoduodenal (\#12), common hepatic artery (\#8), retropancreatic (\#13), and para-aortic (\#16). T-tubes were routinely inserted into the common bile duct. As noted above, liver resection was not performed for all $\mathrm{T} 1$ and $\mathrm{T} 2$ lesions. If the frozen biopsy results were negative or indeterminate, no further operation was performed until the pathology was confirmed. If the results confirmed a T1b or T2 lesion, radical lymph node dissection was subsequently performed. Cases with $\mathrm{Tl} b$ and $\mathrm{T} 2$ lesions referred from other hospitals after laparoscopic cholecystectomy underwent reoperation via an open approach.

The role of adjuvant chemotherapy remains unclear, with some reports of benefit in node-positive GBC or Rl disease., Therefore, adjuvant chemotherapy was only considered in cases of lymph node metastasis or gallbladder perforation during operation. Postoperative complications were graded according to Clavien-Dindo classifications. ${ }^{9}$

\section{Statistical analysis}

All statistical analyses were performed using Statistical Package for Social Sciences version 20 (IBM Corp., Armonk, NY, USA). For each quantitative variable, Shapiro-Wilk test was used as a test of normality. Disease-specific survival and disease-free survival were analyzed using Kaplan-Meier method and compared by log-rank tests. Cox proportional hazards model was used for multivariate survival analysis. Statistical significance was set as $p$ value $<0.05$. 


\section{RESULTS}

\section{Clinicopathologic characteristics}

For the 109 patients included in our analysis, the mean age was $65 \pm 9$ years, and 47 patients ( $43 \%$ ) were male. Forty-five patients (41\%) were diagnosed with incidental GBC. Among them, 11 patients (24\%) were diagnosed with Tla GBC and did not undergo further operations. The mean level of carbohydrate antigen $19-9$ was $13.0 \pm 16.8 \mathrm{U} / \mathrm{mL}$. The median followup was 50 months (range, 5-145 months). The mean number of retrieved lymph nodes was $14 \pm 9$ lymph nodes. Node-positive GBC was found in 14 patients (13\%). Among them, 12 patients underwent adjuvant chemotherapy and two patients refused adjuvant therapy.

\section{Comparison of clinicopathologic outcomes between T stages}

Clinicopathologic characteristics according to T stage are shown in Table 1. Clinical characteristics did not differ among $\mathrm{T}$ stages. There were no differences in terms of the locations of tumors, i.e., hepatic vs. peritoneal sides ( $p=0.805)$; however, tumors involving hepatic side were more frequent ( $\mathrm{n}=63,58 \%$ ). With regard to histologic differentiation, higher $\mathrm{T}$ stages were significantly associated with poorly differentiated GBC ( $p=$ 0.004 ). Two patients in T2 group with poorly differentiated GBC showed combined neuroendocrine features in the pathologic report. Significantly higher lymph node metastasis was noted in T2 group than those in T1b and Tla groups (21\% vs. $4 \%$ and $0 \%$, respectively, $p<0.001$ ). The numbers of retrieved lymph nodes were higher for $\mathrm{T} 1 \mathrm{~b}$ and $\mathrm{T} 2$ groups than for Tla group $(p<0.001)$ (Table 1). In Tla group, six patients who were diagnosed with incidental GBC did not undergo lymph node dissection, whereas 15 patients did as a higher T stage was suspected.

There were no significant differences between stages in terms of complications ( $p=0.467)$. All grade I complications were ei-

Table 1. Clinicopathologic Characteristics according to T Stage

\begin{tabular}{|c|c|c|c|c|}
\hline Variables & T1a $(n=21)$ & T1b $(n=26)$ & $T 2(n=62)$ & $p$ value \\
\hline Age (yr) & $62(40-81)$ & $67(35-82)$ & $65(47-81)$ & 0.331 \\
\hline Male & $9(43)$ & $10(38)$ & $28(45)$ & 0.836 \\
\hline CA 19-9 (U/mL) & $10.1(1.2-83.6)$ & $6.7(0.6-51.7)$ & $8.4(0.1-96.8)$ & 0.380 \\
\hline Incidental gallbladder cancer & $11(52)$ & $12(46)$ & $22(35)$ & 0.359 \\
\hline Tumor size $(\mathrm{cm})$ & $2.0(0.1-4.5)$ & $2.0(0.1-4.2)$ & $2.2(0.3-8.5)$ & 0.160 \\
\hline Tumor location & & & & 0.805 \\
\hline Hepatic side & $11(52)$ & $16(62)$ & $36(58)$ & \\
\hline Peritoneal side & $10(48)$ & $10(38)$ & $26(42)$ & \\
\hline Histologic differentiation & & & & 0.004 \\
\hline Well & $19(90)$ & $21(81)$ & $31(50)$ & \\
\hline Moderate & $2(10)$ & $4(15)$ & $23(37)$ & \\
\hline Poor & 0 & $1(4)$ & $8(13)$ & \\
\hline N stage & & & & $<0.001$ \\
\hline $\mathrm{Nx}$ & $6(29)$ & 0 & 0 & \\
\hline NO & $15(71)$ & $25(96)$ & $49(79)$ & \\
\hline N1 & 0 & $1(4)$ & $11(18)$ & \\
\hline N2 & 0 & 0 & $2(3)$ & 0.273 \\
\hline Lymphovascular invasion & 0 & 2 (8) & $9(15)$ & 0.881 \\
\hline Perineural invasion & $1(5)$ & $1(4)$ & $3(5)$ & $<0.001$ \\
\hline Median number of retrieved LNs & $2(0-19)$ & $16(2-28)$ & $18(3-35)$ & \\
\hline Operation method & & & & $<0.001$ \\
\hline Laparoscopic & $16(76)$ & $5(19)$ & $10(16)$ & \\
\hline Robotic & $3(14)$ & $4(15)$ & $5(8)$ & \\
\hline Open & $2(10)$ & $17(65)$ & $47(76)$ & \\
\hline Complications (Clavien-Dindo) & & & & 0.467 \\
\hline I & 0 & $1(4)$ & $7(11)$ & \\
\hline II & $1(5)$ & 0 & $4(6)$ & \\
\hline III & $1(5)$ & 2 (8) & $3(5)$ & \\
\hline Adjuvant therapy & 0 & $1(4)$ & $11(18)$ & 0.028 \\
\hline
\end{tabular}

LN, lymph node.

Values are presented as median (range) or $\mathrm{n}(\%)$ unless otherwise indicated. 


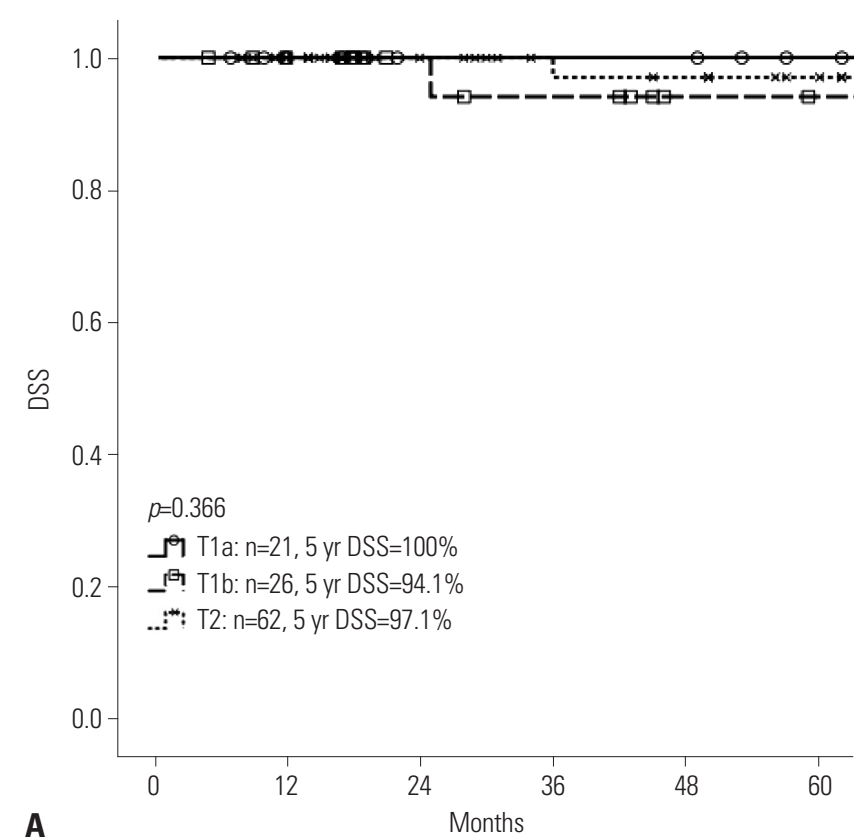

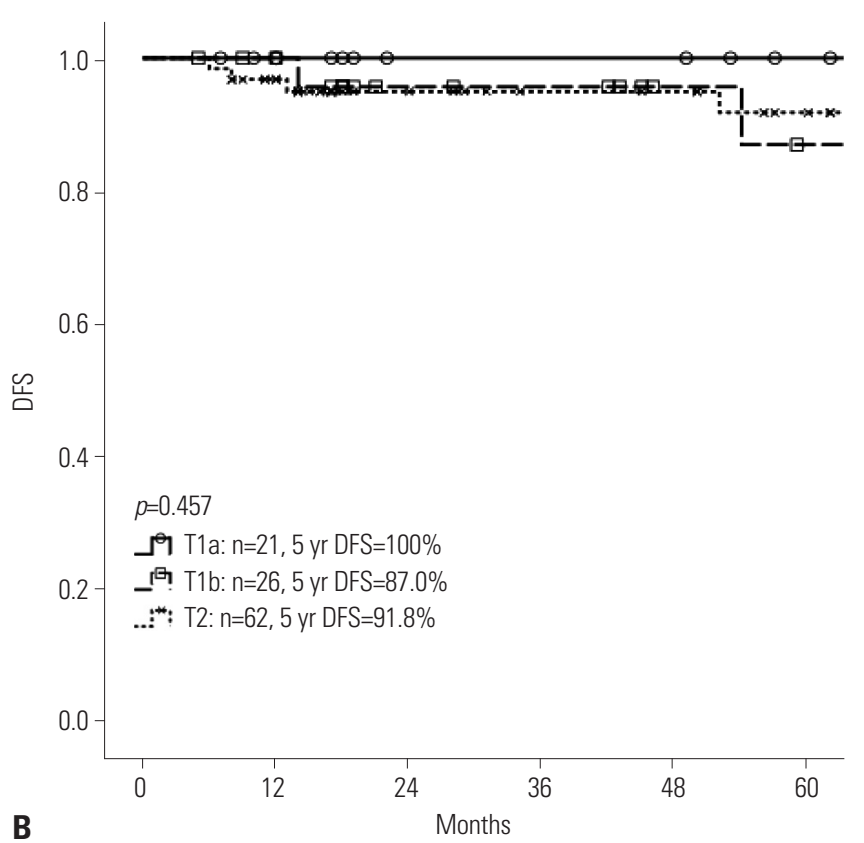

Fig. 2. Oncologic outcomes according to T stage. (A) Disease-specific survival (DSS). (B) Disease-free survival (DFS).

ther fever or wound seroma. Grade II complications were chylous leakage (two cases), which were medically managed, and abscess formation (three cases), which were managed with antibiotics. Grade III complications were postoperative bile leakage requiring endoscopic retrograde biliary drainage (five cases), and intra-abdominal abscess formation requiring percutaneous catheter drainage (one case).

\section{Operative technique and postoperative complications} Open radical cholecystectomies were performed in 66 patients (61\%), whereas the approaches were laparoscopic for 31 patients (28\%) and robotic for 12 patients (11\%). The median (range) operation times for open, laparoscope, and robotic radical cholecystectomies were 183 (95-340), 73 (30-173), and 231 (172-278) $\mathrm{min}$, respectively $(p<0.001)$. There were three cases of open conversion during minimally invasive approaches: one from phrenic artery injury (laparoscopic approach) and two from renal vein injury and aortic wall bleeding (robotic approach).

There were no significant differences in complications between operative techniques ( $p=0.066)$. For minimally invasive approaches, there were no complications in laparoscopic group, but three complications (24\%) were observed in robotic group: grade I for fever, which was treated conservatively; grade II for intra-abdominal abscess formation requiring antibiotics; and grade IIIA requiring endoscopic retrograde biliary drainage due to postoperative bile leakage. There were 13 complications (21\%) in patients undergoing an open approach: six cases were grade I for fever and wound seroma, four in grade II (including two cases of postoperative chylous leakage and two cases of intra-abdominal abscess formation requiring pharmacologic treatments), and three in grade IIIA (including two cases of endoscopic retrograde biliary drainage due to postoperative bile leakage and one case of percutaneous catheter drainage for intra-abdominal abscess formation).

\section{Oncologic outcomes}

There were four GBC-related mortalities during the study period, and the 5-year disease-specific survival rate was $97 \%$. There were no significant differences according to $\mathrm{T}$ stage in disease-specific survival ( $p=0.366$; Fig. $2 \mathrm{~A}$ ) or disease-free survival ( $p=0.457$; Fig. $2 \mathrm{~B}$ ). In all T stages, the median survival was not reached for analysis. In N2 disease, significantly poor oncologic outcomes (5-year disease-specific survival: 50\%, 5-year disease-free survival: 0\%; Fig. 3) were observed. Oncologic outcomes were not associated with the tumor location (Fig. 4) nor the type of surgical approach (Fig. 5).

\section{Recurrence pattern}

The details on recurrence for each GBC stage are shown in Table 2. Overall, there were six cases of recurrence, with a 5-year disease-free survival rate of $92.4 \%$. In cases with Tla GBC, no lymph node metastasis was found in 15 patients who underwent lymph node dissection. Among the 26 patients with T1b lesions, there was only one patient (4\%) with lymph node metastasis, and two patients (8\%) had recurrence. For T2 GBC, 13 patients $(22 \%)$ had lymph node metastasis, two of whom were staged N2: one patient had only single lymph node metastasis in retropancreatic lymph nodes from total of 26 lymph nodes investigated, and the other patient had one metastatic paraaortic lymph node and four metastatic hepatoduodenal lymph nodes from a total of 20 lymph nodes investigated. These patients with N2 staging showed recurrences. Two patients showing poorly differentiated adenocarcinoma with neuroendo- 

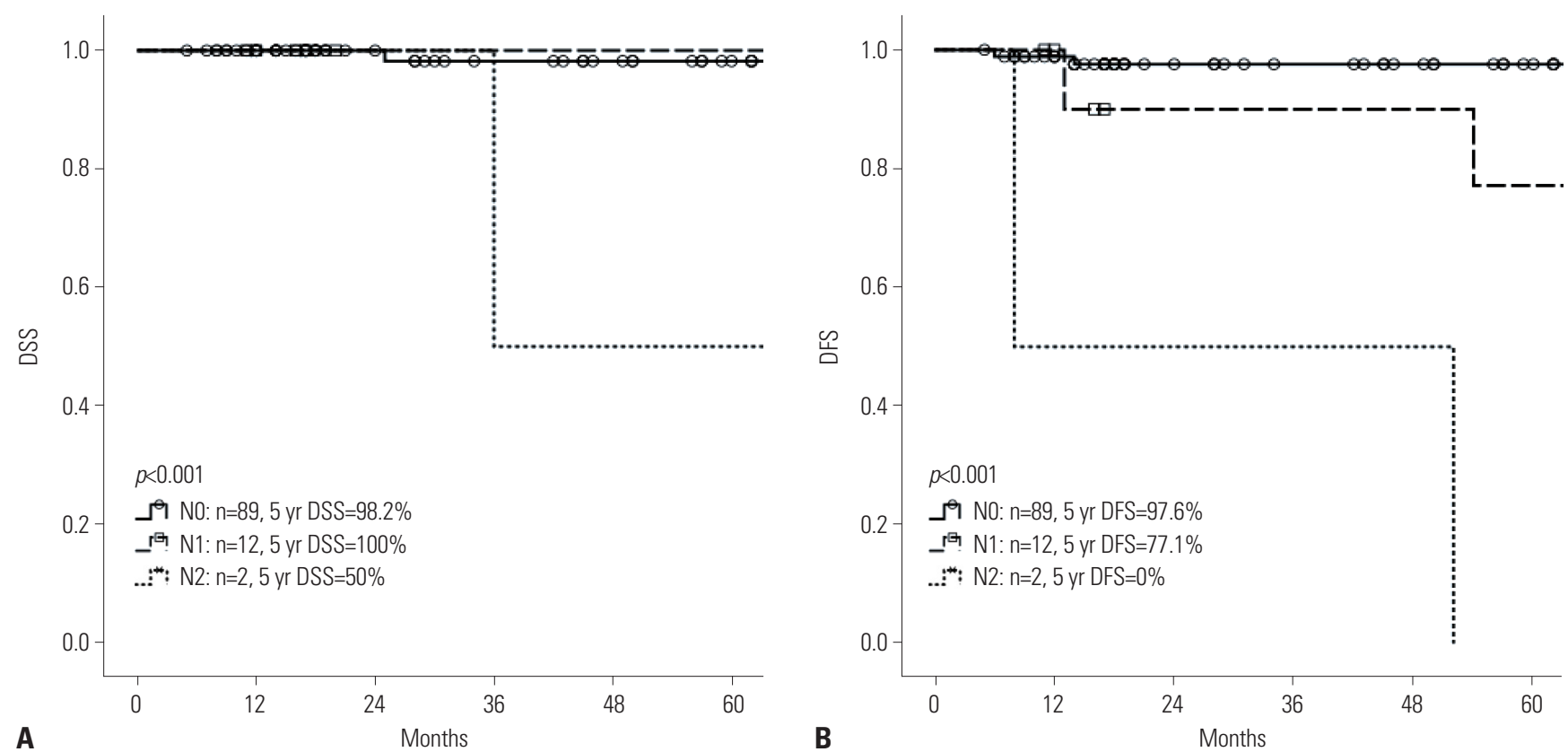

Fig. 3. Oncologic outcomes according to $\mathrm{N}$ stage. (A) Disease-specific survival (DSS). (B) Disease-free survival (DFS).

crine features had recurrence within 1 year after the operation.

\section{DISCUSSION}

Historically, GBC has been associated with pessimistic oncologic outcomes. In 1924, Blalock ${ }^{10}$ wrote that "in malignancy of the gallbladder, when a diagnosis can be made without exploration, no operation should be performed, in as much as it only shortens the patient's life." With improvements in radiology and the invention of laparoscopic operations, early diagnoses of GBC have become more frequent. However, the curative options are limited to radical operations, as GBC has a propensity for early dissemination and is proximal to the liver and major vasculature. Recently, favorable outcomes after less radical cholecystectomies have been reported in patients with T1/T2 GBC, with some reports on the safety and non-inferior oncologic outcomes of laparoscopic approach. ${ }^{11-14}$ However, there is no consensus on the extent of surgery during radical cholecystectomy except for T1a GBC, in which laparoscopic simple cholecystectomy is adequate. Further lymph node dissection may still be indicated, as frozen biopsies during operations cannot always discriminate between T1 and T2 GBC.

Clarification of the oncologic outcomes for GBC is difficult due to the heterogeneity of operative techniques. For example, the extent of surgery differs among surgeons and institutions. Therefore, to compare oncologic outcomes based on the extent of surgery, we analyzed cases involving a single surgeon (WJL) at Severance Hospital, where laparoscopic operations for GBC have been performed since 1993. At that time, if a patient was diagnosed with incidental Tla GBC, routine follow-up was scheduled. For those with T1b GBC or higher, open radical cho- lecystectomies were performed, unless the patients refused for personal reasons. Our previous reports on patients who did not undergo further radical cholecystectomy due to personal reasons or the patients' general condition showed that the 5-year survival rates of GBC patients with $\mathrm{T} 1 \mathrm{a}, \mathrm{T} 1 \mathrm{~b}$, and T2 lesions were $100 \%, 75 \%$, and $56.2 \%$, respectively. ${ }^{15}$ Two cases of recurrences in para-aortic lymph nodes were noted after radical cholecystectomy, including only regional lymphadenectomy, in patients with T2 GBC at 1 month and 5 months. There was no recurrence in the liver bed, which extends from the cystic plate, in any of the patients. Starting in 2005, with the introduction of robotic system at Severance Hospital, minimally invasive radical cholecystectomies, including the robotic approach, were also performed. Our results have shown no differences in oncologic outcomes among different surgical approaches. Therefore, for T1/T2 GBC, having a consistent and reasonable operative principle is more important than the mode of surgical approach.

Our anecdotal experiences have indicated that the dissection of regional lymph nodes along with para-aortic lymph nodes may reduce recurrence. Para-aortic lymph nodes are the final destination in the abdominal lymphatic route from the gallbladder via the cholecysto-retropancreatic pathway and the retroportal node. ${ }^{16}$ However, evidence on the role of extensive lymphadenectomy is lacking. Tsukada, et al. ${ }^{17}$ reported that in T2 GBC, metastases in para-aortic lymph nodes were discovered in $12 \%$ of cases, whereas Ogura, et al. ${ }^{18}$ reported lymph node metastases in $44.3 \%$, with $2.5 \%$ of $\mathrm{Tla}$ and $15.6 \%$ of $\mathrm{Tlb}$ cases also showing metastases. Nevertheless, para-aortic lymphadenectomy did not seem to provide any survival benefit. ${ }^{19}$ In contrast, our findings suggest a benefit of extensive lymphadenectomy on the oncologic outcomes of GBC patients without pre-existing lymph node metastases. 

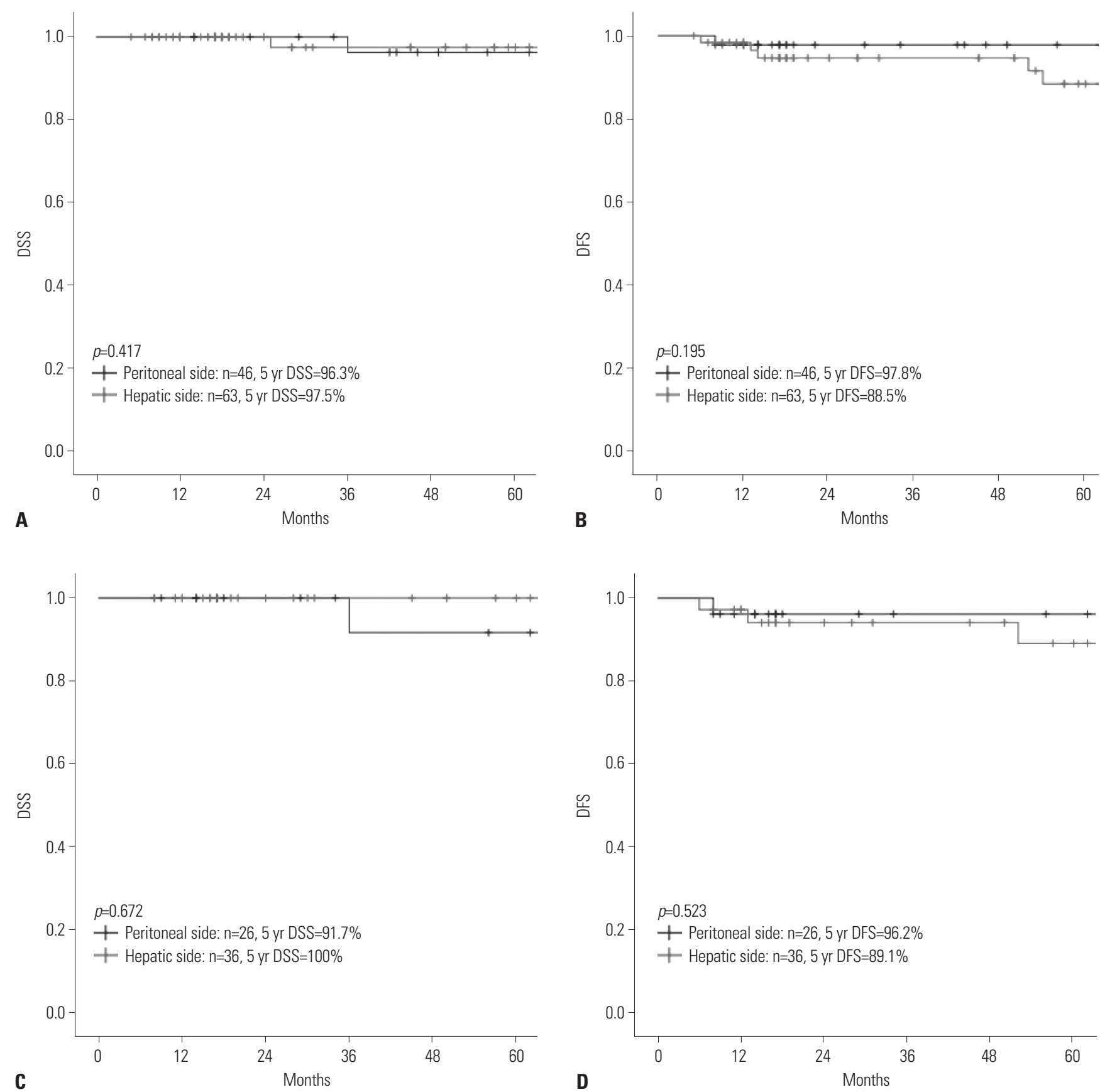

Fig. 4. Oncologic outcomes according to tumor location. (A) Disease-specific survival (DSS). (B) Disease-free survival (DFS). (C) DSS for T2. (D) DFS for T2.

One recent meta-analysis of T1 GBC found comparable survival outcomes between simple and extended cholecystectomies, ${ }^{20}$ whereas another meta-analysis reported conflicting results and suggested an improved prognosis associated with liver resection and lymph node resection for stage T1b GBC or higher. ${ }^{21}$ However, the authors of both meta-analyses noted that a lack of essential data for comparison and a lack of standardization in operative extent and definition can limit the interpretation of the results. Additionally, since liver parenchyme is not involved in T1/T2 GBC, the complete excision of the cystic plate along with the gallbladder may be sufficient. Indeed, our experiences after the implementation of these principles in 2005 have indicated better oncologic outcomes for GBC, especially for T2 tumors. The 5-year disease-specific survival rate was $97.1 \%$ at Severance Hospital, compared to rates ranging from $69.4 \%$ to $90.2 \%$ in previous studies. ${ }^{6,14,22}$ Although this study found one recurrence in the liver, it was bi-lobular and distant from the cystic plate. Horiguchi, et al. ${ }^{23}$ also reported no difference in disease-free survival rates between patients receiving cystic bed resection only and those also undergoing liver resection. Therefore, the complete excision of the cystic plate should be adequate for T1/T2 GBC. 

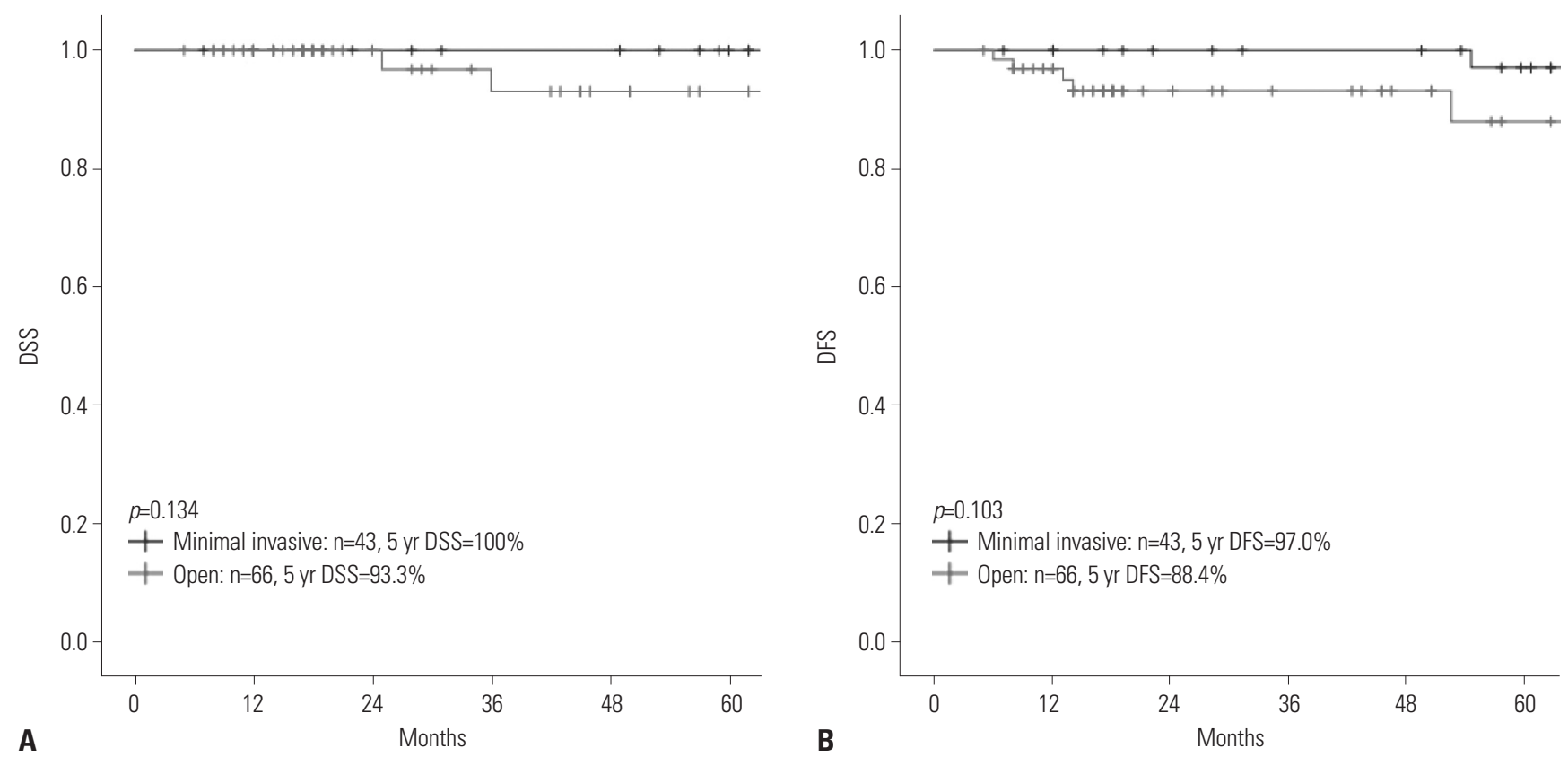

Fig. 5. Oncologic outcomes according to surgical approach. (A) Disease-specific survival (DSS). (B) Disease-free survival (DFS).

Table 2. Lymph Node Status and Recurrence Patterns according to T Stage

\begin{tabular}{|c|c|c|c|c|c|c|}
\hline T stage $(n=112)$ & Nodal status & Number of recurrence & Site of recurrence & Time of recurrence & Survival months & Status \\
\hline \multirow[t]{2}{*}{ T1a $(n=21)$} & $N x^{*}(n=6,29 \%)$ & None & - & & & \\
\hline & NO $(n=15,71 \%)$ & None & - & & & \\
\hline \multirow[t]{2}{*}{$\mathrm{T} 1 \mathrm{~b}(\mathrm{n}=26)$} & NO (n=25, 96\%) & $1(4 \%)$ & Para-aortic LN & 14 & 25 & Dead \\
\hline & N1 (n=1,4\%) & $1(100 \%)$ & Spine & 53 & 60 & Dead \\
\hline \multirow[t]{4}{*}{$\mathrm{T} 2$ (n=62) } & NO (n=49, 79\%) & $1(2 \%)$ & Liver $^{\ddagger}$ & 6 & 20 & Alive \\
\hline & $\mathrm{N} 1(\mathrm{n}=11,18 \%)$ & $1(9 \%)$ & Regional LN, Supraclavidular LN & 13 & 15 & Alive \\
\hline & $\mathrm{N} 2^{\dagger}(\mathrm{n}=2,3 \%)$ & $2(100 \%)$ & Common bile duct & 52 & 65 & Dead \\
\hline & & & Paraortic LN LN $^{\ddagger}$ & 8 & 36 & Dead \\
\hline
\end{tabular}

LN, lymph node.

${ }^{*} \mathrm{Nx}$ represents without lymph node dissection for examination; ${ }^{\dagger} \mathrm{N} 2$ represents positive para-aortic lymph nodes; ${ }^{\sharp}$ Histologic grade showed poorly differentiated adenocarcinoma with neuroendocrine features.

Nortably, the percentage of incidental GBC cases following cholecystectomy in the present report was higher than those reported for laparoscopic cholecystectomies, which ranged from $0.2 \%$ to $1.1 \%{ }^{24}$ This may be due to the fact that Severance Hospital is a tertiary referral hospital. Additionally, our results indicate that the location of the tumor, whether on the hepatic or peritoneal side, does not influence the oncologic outcome, even though the current 8th AJCC staging system further divides $\mathrm{T} 2$ stage accordingly.

In terms of recurrence, six incidences were observed in our study group. Two cases of recurrence showed that GBC was poorly differentiated with neuroendocrine features, which is known to behave more aggressively. ${ }^{25}$ Among the remaining four cases, one patient was initially diagnosed with Tlb lesion without lymph node metastasis, and had recurrence at the paraaortic lymph node 14 months later. This may have resulted from an incomplete para-aortic lymph node dissection; therefore, radical cholecystectomy in Tlb should be sought more actively.

Regarding postoperative complications, some patients showed postoperative bile leakage. These complications may have resulted from complete and radical dissections of hepatoduodenal lymph nodes, which completely expose the common bile duct and disrupt its blood supply. We now routinely insert T-tubes to prevent such postoperative biliary complications.

The limitations of the present study include the limited number of patients analyzed, as well as the inherent selection bias resulting from the retrospective design and selection criteria of the study. Five patients who underwent additional hepatectomy under the suspicion of T3 tumor were excluded from this study. The operative finding showed that the possibility of acute inflammation and liver invasion could not be completely excluded. Therefore, the current treatment algorithm should be applied with discretion in patients with acute inflammation. Furthermore, these results were based on the experience of a 
single surgeon (WJL). Nevertheless, the preventive role of extensive lymphadenectomy, including para-aortic lymph node dissection, should be given further attention, and more surgeons should consider our operative principle to enable a largescale cohort study.

A complete $\mathrm{R} 0$ resection in GBC is the standard of care in patients with localized disease. However, there is no consensus on the extent of radical cholecystectomy for T1/T2 GBC, partly due to the low incidence of GBC that impedes randomized controlled trials to establish optimal treatment modalities. Here, we report on our experiences with an operative principle at Severance Hospital, which indicates that extended lymphadenectomy with para-aortic lymph node dissection without liver resection for T1/T2 GBC produces favorable oncologic outcomes.

\section{AUTHOR CONTRIBUTIONS}

Conceptualization: Woo Jung Lee. Data curation: All authors. Formal analysis: Jae Uk Chong. Funding acquisition: Woo Jung Lee. Investigation: All authors. Methodology: All authors. Project administration: Woo Jung Lee. Resources: All authors. Software: All authors. Supervision: Woo Jung Lee. Validation: All authors. Visualization: All authors. Writing_original draft: All authors. Writing_review \& editing: All authors.

\section{ORCID iDs}

Jae Uk Chong https://orcid.org/0000-0001-9713-1653

Woo Jung Lee https://orcid.org/0000-0001-9273-261X

\section{REFERENCES}

1. Hueman MT, Vollmer CM Jr, Pawlik TM. Evolving treatment strategies for gallbladder cancer. Ann Surg Oncol 2009;16:2101-15.

2. Lazcano-Ponce EC, Miquel JF, Muñoz N, Herrero R, Ferrecio C, Wistuba II, et al. Epidemiology and molecular pathology of gallbladder cancer. CA Cancer J Clin 2001;51:349-64.

3. Lee SE, Kim KS, Kim WB, Kim IG, Nah YW, Ryu DH, et al. Practical guidelines for the surgical treatment of gallbladder cancer. J Korean Med Sci 2014;29:1333-40.

4. Randi G, Franceschi S, La Vecchia C. Gallbladder cancer worldwide: geographical distribution and risk factors. Int J Cancer 2006;118:1591-602.

5. Lee SE, Jang JY, Lim CS, Kang MJ, Kim SW. Systematic review on the surgical treatment for T1 gallbladder cancer. World J Gastroenterol 2011;17:174-80.

6. Kim DH, Kim SH, Choi GH, Kang CM, Kim KS, Choi JS, et al. Role of cholecystectomy and lymph node dissection in patients with T2 gallbladder cancer. World J Surg 2013;37:2635-40.

7. Hoehn RS, Wima K, Ertel AE, Meier A, Ahmad SA, Shah SA, et al. Adjuvant therapy for gallbladder cancer: an analysis of the National Cancer Data Base. J Gastrointest Surg 2015;19:1794-801.

8. Ma N, Cheng H, Qin B, Zhong R, Wang B. Adjuvant therapy in the treatment of gallbladder cancer: a meta-analysis. BMC Cancer 2015;15:615.

9. Dindo D, Demartines N, Clavien PA. Classification of surgical complications: a new proposal with evaluation in a cohort of 6336 patients and results of a survey. Ann Surg 2004;240:205-13.

10. Blalock A. A statistical study of eight hundred and eighty-eight cases of biliary tract disease. Johns Hopkins Hosp Bull 1924;35: 391-409.

11. Itano O, Oshima G, Minagawa T, Shinoda M, Kitago M, Abe Y, et al. Novel strategy for laparoscopic treatment of pT2 gallbladder carcinoma. Surg Endosc 2015;29:3600-7.

12. Lee H, Choi DW, Park JY, Youn S, Kwon W, Heo JS, et al. Surgical strategy for T2 gallbladder cancer according to tumor location. Ann Surg Oncol 2015;22:2779-86.

13. Zimmitti G, Manzoni A, Guerini F, Ramera M, Bertocchi P, Aroldi F, et al. Current role of minimally invasive radical cholecystectomy for gallbladder cancer. Gastroenterol Res Pract 2016. Article ID: 7684915

14. Yoon YS, Han HS, Cho JY, Choi Y, Lee W, Jang JY, et al. Is laparoscopy contraindicated for gallbladder cancer? A 10-year prospective cohort study. J Am Coll Surg 2015;221:847-53.

15. Kang CM, Lee WJ, Choi GH, Kim JY, Kim KS, Choi JS, et al. Does "clinical" R0 have validity in the choice of simple cholecystectomy for gallbladder carcinoma? J Gastrointest Surg 2007;11:1309-16.

16. Ito M, Mishima Y. Lymphatic drainage of the gallbladder. J Hepatobiliary Pancreat Surg 1994;1:302-8.

17. Tsukada K, Hatakeyama K, Kurosaki I, Uchida K, Shirai Y, Muto T, et al. Outcome of radical surgery for carcinoma of the gallbladder according to the TNM stage. Surgery 1996;120:816-21.

18. Ogura Y, Mizumoto R, Isaji S, Kusuda T, Matsuda S, Tabata M. Radical operations for carcinoma of the gallbladder: present status in Japan. World J Surg 1991;15:337-43.

19. Kondo S, Nimura Y, Hayakawa N, Kamiya J, Nagino M, Uesaka K. Regional and para-aortic lymphadenectomy in radical surgery for advanced gallbladder carcinoma. Br J Surg 2000;87:418-22.

20. Lee H, Kwon W, Han Y, Kim JR, Kim SW, Jang JY. Optimal extent of surgery for early gallbladder cancer with regard to long-term survival: a meta-analysis. J Hepatobiliary Pancreat Sci 2018;25: 131-41.

21. Sternby Eilard M, Lundgren L, Cahlin C, Strandell A, Svanberg T, Sandström P. Surgical treatment for gallbladder cancer-a systematic literature review. Scand J Gastroenterol 2017;52:505-14.

22. Jung W, Jang JY, Kang MJ, Chang YR, Shin YC, Chang J, et al. Effects of surgical methods and tumor location on survival and recurrence patterns after curative resection in patients with $\mathrm{T} 2$ gallbladder cancer. Gut Liver 2016;10:140-6.

23. Horiguchi A, Miyakawa S, Ishihara S, Miyazaki M, Ohtsuka M, Shimizu H, et al. Gallbladder bed resection or hepatectomy of segments $4 \mathrm{a}$ and 5 for pT2 gallbladder carcinoma: analysis of Japanese registration cases by the study group for biliary surgery of the Japanese Society of Hepato-Biliary-Pancreatic Surgery. J Hepatobiliary Pancreat Sci 2013;20:518-24.

24. Pitt SC, Jin LX, Hall BL, Strasberg SM, Pitt HA. Incidental gallbladder cancer at cholecystectomy: when should the surgeon be suspicious? Ann Surg 2014;260:128-33.

25. Eltawil KM, Gustafsson BI, Kidd M, Modlin IM. Neuroendocrine tumors of the gallbladder: an evaluation and reassessment of management strategy. J Clin Gastroenterol 2010;44:687-95. 